\title{
LA PHILOLOGIE ROMANE EN POLOGNE MÉRITE-T-ELLE ENCORE SON NOM?
}

\section{LA PHILOLOGIE DANS LA NOMENCLATURE UNIVERSITAIRE POLONAISE}

La philologie désigne dans la nomenclature universitaire polonaise deux domaines connexes : les études universitaires des langues et lettres modernes et une discipline de recherche qui en constitue la base. Toutefois, dans l'usage courant, la philologie universitaire est uniquement associée aux études de langues à un niveau hautement spécialisé. Cela n'est pas sans conséquence pour l'image courante de ces études par rapport à leur réel contenu et l'organisation interne ; c'est ce que nous nous proposons de discuter dans le présent article.

Résumons les faits, bien connus par le passé. Dans la tradition humaniste remontant à la Renaissance la philologie est l'étude des textes littéraires et philosophiques de l'Antiquité grecque et latine, ainsi que la description grammaticale des langues classiques entreprise à des fins tant théoriques que didactiques, servant à l'enseignement scolaire. Au XIX ${ }^{\mathrm{e}}$ siècle l'objet de la philologie change, son intérêt est centré sur les langues issues du latin — la Romania — dans leur évolution historique. Puis la recherche s'élargit vers les origines des langues européennes (la famille indo-européenne) grâce à quoi émerge une nouvelle discipline, la grammaire comparée et la typologie des langues européennes. La recherche historique porte aussi bien sur les familles européennes : langues germaniques, slaves, baltes, que sur les langues orientales; elle jette ainsi les bases pour les études universitaires des langues modernes qui, tributaires de leur source, prennent le nom de philologie, avec l'épithète spécifiant le groupe de langues qui constitue leur objet: 
philologie romane, slave, germanique, classique, orientale. Cette dénomination a été généralement adoptée dans les universités européennes au XIX ${ }^{\mathrm{e}}$ siècle. En Pologne, notamment dans le dernier quart du $\mathrm{XX}^{\mathrm{e}}$ siècle, les études des langues et lettres occidentales modernes prennent le nom générique de neofilologia qui regroupe ces disciplines de manière purement administrative ; par exemple, à côté des familles historiques comme filologia romańska, filologia germańska, apparaît filologia angielska en tant qu'unité à part. Ensuite, avec une spécialisation limitant le cursus à l'étude d'une seule langue, dans la nomenclature universitaire polonaise (probablement sous l'influence de la terminologie allemande) apparaissent, pour la presque totalité des branches philologiques, des noms spécifiant leur objet : italianistyka, rusycystyka, polonistyka, anglistyka, mais aussi iberystyka, skandynawistyka, amerykanistyka, etc. La même tendance s'étend à la philologie orientale ou orientalistyka qui éclate en arabistyka, japonistyka, mongolistyka et d'autres, selon la langue et la culture étudiées.

La diversification croissante du contenu des spécialisations en langues et lettres modernes ne va pas de pair avec la nomenclature des diplômes universitaires polonais qui doivent toujours porter l'intitulé filologia. Il y a donc une sérieuse raison d'examiner le rapport entre le contenu actuel des études universitaires de langues et lettres et ce titre riche d'une longue et illustre tradition. Par ailleurs, il peut prêter à confusion, car dans la nomenclature scientifique française la philologie désigne uniquement l'étude linguistique et historique des textes anciens, dans le but de préparer leur édition critique.

\section{LA PHILOLOGIE ROMANE EN FRANCE - SITUATION ACTUELLE}

Nous trouvons intéressant de citer à ce propos l'article, relativement récent, de Frédéric Duval ${ }^{1}$. L'idée maîtresse de l'article est de démontrer l'importance de la philologie romane pour les sciences humaines d'aujourd'hui. Son auteur professeur de philologie romane à l'École des chartes - plaide pour préserver le « patrimoine écrit » contre « une course effrénée vers la modernité » dont l'un des effets est la marginalisation des sciences humaines. La philologie dont l'objet est l'étude et l'édition des textes et documents anciens préserve de la rupture avec la tradition culturelle et permet d'approcher l'univers intellectuel d'autrefois tout en montrant son altérité ${ }^{2}$.

La situation actuelle de la philologie romane en France suscite des polémiques concernant sa place dans la hiérarchie des sciences humaines modernes.

${ }^{1}$ F. Duval, « À quoi sert encore la philologie ? Politique et philologie aujourd'hui », Laboratoire italien. Politique et société, $\mathrm{n}^{\mathrm{0}} 7 / 2007$ : Philologie et politique, pp. 17-40, <https:// laboratoireitalien.revues.org> [consulté le 28.07.2017].

2 Ibidem, p. 17. 
Considérée comme une discipline utilisant des méthodes surannées, centrée sur une exégèse pédante des anciens textes, " la philologie fut rapidement absorbée par des départements de langue et de littérature $»^{3}$. Son image internationale en a souffert à tel point que, selon Duval « une enquête menée auprès d'universitaires américains a montré qu'une partie d'entre eux ignoraient ce qu'était la philologie et que les autres la considéraient comme une branche "obsolète" de la linguistique $»^{4}$.

Contrairement aux critiques qui la marginalisent, Duval voit la philologie comme une discipline complexe, réunissant les études linguistiques, littéraires et historiques au service de la démarche herméneutique appliquée à des documents écrits qui, pour être édités, nécessitent tout un appareil explicatif relevant des sciences humaines. Les « contours flous » de la philologie sont à la fois son handicap et sa force, car elle peut, en retour, offrir la richesse de ses recherches à la linguistique cognitive, la lexicologie, les études littéraires et l'histoire. En même temps, les technologies numériques mises au service de la philologie permettent d'ajourner et de rénover ses traditionnelles méthodes de travail.

En concluant sa défense et illustration de la philologie, Duval voit dans cette discipline un remède contre la tendance à une modernisation irréfléchie :

Les tentatives visant à faire entrer la philologie dans la modernité tiennent d'une certaine façon du « bougisme » : la " nouvelle philologie », supérieure parce que nouvelle, s'est ainsi opposée à la « vieille philologie » et aux dinosaures. La résistance à la " nouvelle philologie » a prouvé que l'essentiel restait le texte et non l'habillage théorique qui permettrait de mettre la discipline à la mode. Défendre la philologie — ni vieille ni moderne — c'est défendre la liberté d'accéder aux connaissances ; c'est également affranchir la connaissance scientifique des impératifs utilitaristes ${ }^{5}$.

Telle est, selon un philologue de notre temps, la situation actuelle de cette discipline scientifique en France. Cela se reflète dans l'organisation des facultés de langues et lettres dans les universités françaises. Les établissements qui ont dans leur cursus une composante philologique portent habituellement le nom de départements ou chaires d'études françaises, la philologie romane n'y apparaît pas, probablement pour des raisons de difficulté terminologique, résultant de l'autonomie des études d'autres langues romanes. La spécialisation se manifeste jusque dans la nomenclature universitaire ; les diplômes universitaires des disciplines jadis « philologiques » s'intitulent lettres modernes, études hispaniques, études italiennes, linguistique, etc., jusqu'à l'École des chartes où est décerné, parmi d'autres, un diplôme d'études médiévales.

\footnotetext{
${ }^{3}$ Ibidem, p. 19.

${ }^{4}$ Ibidem.

${ }^{5}$ Ibidem, p. 36.
} 


\section{LA PHILOLOGIE ROMANE EN POLOGNE : QUELQUES REPÈRES HISTORIQUES}

La philologie romane dans les universités polonaises a une tradition plus que centenaire $^{6}$. Historiquement, l'objet de la philologie romane comprend toutes les langues issues du latin ainsi que l'histoire de la littérature où une place de choix est réservée à l'époque médiévale. Toutefois, grâce au prestige dont jouissait la langue française dans les milieux cultivés de la société polonaise d'autrefois, l'intérêt porté à cette langue dépassait de loin celui consacré aux autres idiomes de la Romania. Il est à noter aussi que le français occupait une place de choix parmi les thèmes de recherches philologiques, surtout en France et en Allemagne.

Ainsi, les premières chaires universitaires de philologie romane (Cracovie 1892, Lvov 1894, Varsovie 1919, Poznań 1919) réservaient dans leur cursus d'études une place importante à la grammaire historique des langues romanes et à la littérature française, enseignée dans une perspective chronologique. Une autre langue romane, l'italien ou l'espagnol, complétait le cursus. Le latin étant une matière majoritairement enseignée dans le secondaire (jusqu'au baccalauréat), l'évolution des langues romanes ne posait pas aux étudiants de problèmes d'assimilation majeurs. Il existe un ouvrage de toute première importance qui permet de se rendre compte de l'ampleur des études romanes en Pologne dès la création des chaires de philologie : c'est le livre de Jan Goldman (1907-1942) qui contient une bibliographie raisonnée des travaux parus jusqu'aux années trente du $\mathrm{XX}^{\mathrm{e}}$ siècle ${ }^{7}$.

Après 1945 les quelques chaires polonaises de philologie romane n'avaient qu'une activité restreinte : peu d'étudiants, pratiquement pas de contacts avec les universités européennes, bibliothèques dont les collections s'étaient arrêtées en 1939 au plus tard. Seul avantage pour le français : en Silésie et aux confins ouest du pays, dans les régions attribuées à la Pologne d'après guerre suite aux accords de Yalta et de Téhéran, l'enseignement scolaire de l'allemand était pratiquement absent ${ }^{8}$, l'anglais — langue des impérialistes américains — décidément mal vu dans tout le système éducatif, alors le français comme deuxième langue moderne (après le russe) s'imposait, au choix avec le traditionnel latin. Et c'est peut-être grâce à ce concours de circonstances que les études de philologie romane ont pu

${ }^{6}$ K. Kasprzyk, « Filologia romańska », [dans :] Historia nauki polskiej, t. 4, vol. 3 : 18631918, B. Suchodolski (dir.), Zakład Narodowy im. Ossolińskich — Wydawnictwo Polskiej Akademii Nauk, Wrocław 1987, pp. 771-785.

7 J. Goldman, « La philologie romane en Pologne », Archivum Neophilologicum 2, Kraków 1937. Note sur l'auteur : S. Rospond, « Jan Goldman », Pamiętnik Literacki 36/1-2, 1946, pp. 165166, <http://bazhum.muzhp.pl/czasopismo/8/ ?idno=2112> [consulté le 25.07.2017].

${ }^{8}$ P. Baron, « Nauczanie języka niemieckiego jako języka mniejszości na Opolszczyźnie », [dans :] Colloquium Opole 2007. Mniejszości narodowe i etniczne - edukacja i kultura, S. Senft, A. Trzcielińska-Polus (dir.), Państwowy Instytut Naukowy — Instytut Śląski w Opolu, Opole 2008, pp. 76-88. 
survivre, d'autant plus qu'on leur avait ajouté une composante pédagogique obligatoire, afin de former des professeurs de français.

Dans les années soixante du $\mathrm{XX}^{\mathrm{e}}$ siècle les études historiques sont concurrencées par une discipline qui, proscrite par la censure des années cinquante comme dangereuse pour le matérialisme dialectique, peut regagner la place qui lui était due : c'est le structuralisme linguistique ${ }^{9}$, d'ailleurs il est bientôt suivi par la linguistique générative. Cela change progressivement les proportions entre la linguistique historique et les nouvelles méthodologies qui se montrent plus efficaces dans certains secteurs de recherche sur les langues.

\section{LES PROGRAMMES D'ÉTUDES DES DÉPARTEMENTS DE PHILOLOGIE ROMANE ONT SUBI UNE PROFONDE TRANSFORMATION}

En Pologne, un changement réduisant l'importance de la philologie comme matière du cursus universitaire est survenu pendant les trente dernières années. Les circonstances qui l'avaient accéléré sont multiples, notamment :

- un grand essor de nouveaux domaines de recherche en linguistique, indépendants du fond historique de la langue (cognitivisme, analyse du discours, linguistique computationnelle, grands corpus électroniques);

- dans l'enseignement scolaire des langues, une place de plus en plus réduite du français par rapport à l'anglais, l'allemand et autres langues modernes ;

- un marché du travail en constante évolution, défavorisant les traditionnelles professions des philologues comme professeur de français, traducteur de la littérature ou éditeur dans une maison d'édition;

— enfin, l'attrait de la civilisation française qui va diminuant par rapport à l'omniprésente pop-culture d'inspiration américaine.

Les départements philologiques universitaires ne sont pas restés indifférents au défi que présentait pour leur fonctionnement la nouvelle situation politique et sociale en Pologne. Déjà en 1993, un colloque international tenu à l'Institut d'études romanes de l'Université de Varsovie réunissait des intervenants de huit pays autour du thème du français langue étrangère à l'université ${ }^{10}$. Le renouveau des études philologiques devait selon les intervenants commencer par de nouvelles méthodes d'enseignement du français : ouverture vers les stratégies communicatives, nouvelles propositions pour l'enseignement de la grammaire, introduction des langues

${ }^{9}$ C'est à cette époque qu'est publiée la traduction en polonais du Cours de linguistique générale: F. de Saussure, Kurs językoznawstwa ogólnego, trad. K. Kasprzyk, PWN, Warszawa 1961 [nouvelles éditions en 1992 et 2002].

${ }^{10}$ Le français langue étrangère à l'université, théorie et pratique. Actes du colloque international, Université de Varsovie, 25-26 novembre 1993, A. Cataldi, K. Zaleska (dir.), Instytut Romanistyki UW, Warszawa 1994. 
de spécialité (en premier lieu langue des affaires) avec la traduction spécialisée, enfin la francophonie comme composante interculturelle. Ces nouvelles propositions devaient, d'une part, réorganiser la didactique du français dans les départements universitaires et de l'autre, former les professeurs de langue capables de se servir de nouvelles méthodes d'enseignement dans les classes du primaire et du secondaire.

Un échange d'idées entre les enseignants et chercheurs universitaires était rendu encore plus fructueux grâce à l'Association académique des romanistes polonais «Plejada », fondée en 2002. Sous ses auspices, un projet intitulé Philologies romanes et orientation professionnelle des étudiants a pu être réalisé dans les années $2004-2005^{11}$. L'enquête, menée dans le cadre du projet, à laquelle avaient répondu 563 récents diplômés en philologie romane, a montré que déjà à cette époque moins que la moitié d'entre eux travaillaient comme professeurs de langue dans des établissements divers, les autres, tout en affirmant l'utilité des connaissances acquises pendant leurs études, exerçaient une autre profession. Deux débats consacrés aux résultats de l'enquête apportaient de nouvelles propositions concernant le programme d'études, entre autres celle de «cultural studies » qui « se rapporte aux nouveaux médias, aux nouvelles formes textuelles et médiales, aux nouveaux discours sociaux et peut déboucher sur d'autres spécialisations professionnelles que celle des enseignants $»^{12}$. Dans le cadre du même débat, l'article de Monika Grabowska montrait comment la philologie dont l'objet est l'analyse critique du texte pourrait servir à développer la compétence à l'étude des textes contemporains de toute nature : narratifs, explicatifs, argumentatifs (et non seulement des textes anciens) chez les étudiants d'aujourd'hui ${ }^{13}$. La conclusion de son article montre comment l'auteur voit la fonctionnalité de la méthode philologique :

Du point de vue d'une meilleure adaptation des étudiants de la faculté au marché du travail, les ressortissants de la philologie romane ayant bien maîtrisé la compétence textuelle seraient bien préparés à tout poste impliquant la production d'un texte et par cela même la communication. [...] Leur atout sera alors la capacité d'une argumentation rigoureuse, d'une démarche réflexive, d'une ouverture vers d'autres disciplines, d'une connaissance de la vie socio-économique et politique actuelle et d'une facilité d'adaptation à d'autres cultures $[\ldots]^{14}$.

Il serait difficile de contester la justesse de cette argumentation, même si la vision du résultat paraît, à l'heure présente, quelque peu idéaliste.

${ }^{11}$ Cf. T. Tomaszkiewicz, « Avant propos », [dans :] Vers une nouvelle dimension des études romanes, T. Tomaszkiewicz (dir.), Oficyna Wydawnicza LEKSEM, Łask 2006, pp. 7-12.

12 Ibidem, p. 10.

${ }^{13}$ M. Grabowska, «La compétence textuelle en philologie romane », [dans :] Vers une nouvelle dimension..., pp. 103-114.

14 Ibidem, p. 113. 


\section{ORGANISATION ACTUELLE DES ÉTABLISSEMENTS «PHILOLOGIQUES »}

Une relative liberté dans l'élaboration des programmes universitaires, ainsi que la nécessité de s'adapter au contexte social dans lequel les jeunes cherchent un profil d'études supérieures correspondant à leurs ambitions, ont encouragé les établissements des langues et lettres modernes à rechercher une nouvelle formule de leurs cursus avec l'abandon progressif des matières philologiques traditionnelles. Ce profond remaniement a pratiquement détruit l'image unitaire des études philologiques universitaires qui étaient censées assurer, dans des proportions égales, l'enseignement pratique d'une seule langue étrangère, un cours d'histoire de la littérature, un cours de linguistique théorique et une spécialisation obligatoire en didactique de cette langue. Toutefois, l'intitulé de philologie n'a pas disparu de la nomenclature ministérielle des disciplines de recherche ni du diplôme universitaire.

Dans ce qui suit nous présentons le résultat d'une brève investigation sur les appellations que s'attribuent actuellement les établissements universitaires des langues et littératures romanes. À cet effet nous avons consulté les sites officiels de ces établissements. La première sélection a porté sur les unités qui conservent leur intitulé de philologie romane (filologia romańska). Ensuite nous avons délimité les appellations romanistyka, italianistyka, hispanistyka et autres de ce type que nous rendons en traduction comme études romanes, études italiennes, études espagnoles, etc., puis celles qui ont dans l'intitulé język francuski - langue française, en terminant par les intitulés divers. En ce qui concerne les noms des structures universitaires nous nous limitons à leur traduction littérale, évitant le terme département qui nous semble inapproprié aux structures universitaires polonaises ${ }^{15}$. Notons enfin que l'appellation polonaise romanistyka que nous rendons par études romanes correspond, en général, aux seules études françaises.

Tableau 1. Instytut Filologii Romańskiej (Institut de philologie romane)

\begin{tabular}{|l|l|}
\hline \multicolumn{1}{|c|}{ Université } & \multicolumn{1}{c|}{ Filières avec un cursus autonome } \\
\hline Université de Gdańsk (UG) & $\begin{array}{l}\text { Philologie romane - seulement pour le français } \\
\text { Études ibériques }\end{array}$ \\
\hline Université Jagellonne, Cracovie (UJ) & $\begin{array}{l}\text { Philologies : française (romane), italienne, es- } \\
\text { pagnole, portugaise, roumaine, avec plusieurs } \\
\text { spécialisations }\end{array}$ \\
\hline
\end{tabular}

${ }^{15}$ Le terme apparaît dans d'autres contextes au sens générique de « unité administrative d'une certaine autonomie au sein d'une structure universitaire ». 


\begin{tabular}{|l|l|}
\hline $\begin{array}{l}\text { Université Marie Curie-Skłodowska, Lublin } \\
\text { (UMCS) }\end{array}$ & $\begin{array}{l}\text { Études françaises et italiennes / italiennes et fran- } \\
\text { çaises } \\
\text { Dans la filière d'études ibériques : études espa- } \\
\text { gnoles, études portugaises } \\
\text { (le tout avec spécialisation en traduction et en } \\
\text { langues de spécialité) }\end{array}$ \\
\hline Université catholique de Lublin (KUL) & $\begin{array}{l}\text { Études françaises (avec l'italien ou l'espagnol } \\
\text { comme 2e langue) } \\
\text { Études espagnoles (avec le français comme 2e } \\
\text { langue) }\end{array}$ \\
\hline $\begin{array}{l}\text { Université Adam Mickiewicz de Poznań } \\
\text { (UAM) }\end{array}$ & $\begin{array}{l}\text { Philologies : romane (i.e. française), italienne, es- } \\
\text { pagnole, portugaise } \\
\text { Le cursus de chaque filière contient une 2e langue } \\
\text { romane. }\end{array}$ \\
\hline Université de Wrocław (UWr) & $\begin{array}{l}\text { Philologies : française, espagnole, études ita- } \\
\text { liennes (en collaboration avec l'Institut d'études } \\
\text { classiques et méditérannéennes) }\end{array}$ \\
\hline
\end{tabular}

Tableau 2. Katedra Filologii Romańskiej (Chaire de philologie romane)

\begin{tabular}{|l|l|}
\hline \multicolumn{1}{|c|}{ Université } & \multicolumn{1}{c|}{ Filières avec un cursus autonome } \\
\hline $\begin{array}{l}\text { Université de Szczecin (USz), Faculté de } \\
\text { philologie }\end{array}$ & $\begin{array}{l}\text { Philologie romane (i.e. française) avec une } 2^{\mathrm{e}} \\
\text { langue }\end{array}$ \\
\hline & $\begin{array}{l}\text { Philologie romane (i.e. française) avec une } 2^{\mathrm{e}} \\
\text { langue } \\
\text { Université Mikołaj Kopernik, Toruń (UMK), } \\
\text { Faculté de philologie } \\
\text { çais-arabe } \\
\text { (NB : Dans la structure de la Faculté de philo- } \\
\text { logie il y a une Chaire de philologie italienne, } \\
\text { indépendante de la Chaire de philologie romane) }\end{array}$ \\
\hline
\end{tabular}

Tableau 3. Divers : neofilologia, filologia romańska, język francuski (néophilologie, philologie romane, langue française)

\begin{tabular}{|l|l|l|}
\hline \multicolumn{1}{|c|}{ Université } & \multicolumn{1}{|c|}{ Nom de l'établissement } & \multicolumn{1}{c|}{$\begin{array}{c}\text { Filière avec un cursus } \\
\text { autonome }\end{array}$} \\
\hline $\begin{array}{l}\text { Université de Białystok } \\
\text { (UwB), Faculté } \\
\text { de philologie }\end{array}$ & $\begin{array}{l}\text { Zakład Języka Francuskiego (Section de } \\
\text { langue française), Instytut Neofilologii } \\
\text { (Institut de néophilologie) }\end{array}$ & $\begin{array}{l}\text { Français et anglais } \\
\text { Français et espagnol } \\
\text { (licence seulement) }\end{array}$ \\
\hline $\begin{array}{l}\text { Université d'Opole } \\
\text { (UO), Faculté de } \\
\text { philologie }\end{array}$ & $\begin{array}{l}\text { Katedra Języka i Kultury Francuskiej } \\
\text { (Chaire de langue et de culture françaises) }\end{array}$ & $\begin{array}{l}\text { Philologie romane } \\
\text { (français seulement) }\end{array}$ \\
\hline
\end{tabular}




\begin{tabular}{|l|l|l|}
\hline & & $\begin{array}{l}\text { Philologie romane } \\
\text { (français seulement) } \\
\text { Philologie romane et } \\
\text { l'anglais } \\
\text { Philologie romane et } \\
\text { le russe } \\
\text { Philologie italienne } \\
\text { Philologie espagnole } \\
\text { de Cracovie (UP) }\end{array}$ \\
\hline $\begin{array}{l}\text { Université de Zielona } \\
\text { Góra (ZG), Faculté des } \\
\text { sciences humaines }\end{array}$ & $\begin{array}{l}\text { Znstytut Neofilologii (Institut de néophi- } \\
\text { lologie) } \\
\text { philologie romane), Instytut Neofilologii } \\
\text { (Institut de Néophilologie) }\end{array}$ & $\begin{array}{l}\text { Français (licence seule- } \\
\text { ment) }\end{array}$ \\
\hline
\end{tabular}

Tableau 4. Instytut Romanistyki (Institut d'études romanes)

\begin{tabular}{|l|l|}
\hline \multicolumn{1}{|c|}{ Université } & \multicolumn{1}{c|}{ Filières avec un cursus autonome } \\
\hline Université de Łódź & $\begin{array}{l}\text { Études françaises } \\
\text { Études italiennes }\end{array}$ \\
\hline \multirow{3}{*}{ Université de Varsovie } & $\begin{array}{l}\text { Études françaises, avec une 2e langue romane ou l'anglais } \\
\text { Études interdisciplinaires (trois langues, dont le français, l'italien, } \\
\text { l'espagnol, l'anglais, l'allemand) en collaboration avec les insti- } \\
\text { tuts de la Faculté de néophilologie }\end{array}$ \\
\hline
\end{tabular}

Tableau 5. Języki romańskie, translatoryka (langues romanes, traduction)

\begin{tabular}{|l|l|}
\hline \multicolumn{1}{|c|}{ Université de Silésie } & \multicolumn{1}{|c|}{ Filières avec un cursus autonome } \\
\hline Instytut Języków & Différentes filières de deux langues combinant le français, l'ita- \\
Romańskich i Translatoryki & lien, l'espagnol et l'anglais dans des programmes consacrés aux \\
(Institut des langues & divers métiers de la traduction. Il existe aussi une filière de phi- \\
romanes et de traduction) & lologie romane. \\
\hline
\end{tabular}

Tableau 6. Lingwistyka stosowana (linguistique appliquée)

\begin{tabular}{|l|l|}
\hline \multicolumn{1}{|c|}{ Université de Varsovie } & \multicolumn{1}{c|}{ Filières avec un cursus autonome } \\
\hline $\begin{array}{l}\text { Instytut Lingwistyki } \\
\begin{array}{l}\text { Stosowanej (Institut de } \\
\text { linguistique appliquée) }\end{array}\end{array}$ & $\begin{array}{l}\text { Filières de deux langues au choix parmi sept : l'anglais, l'alle- } \\
\text { mand, l'espagnol, le français, le japonais, le russe, le suédois }\end{array}$ \\
$\begin{array}{l}\text { Spécialisations : traduction, études interculturelles, linguistique, } \\
\text { didactique des langues étrangères }\end{array}$ \\
\hline
\end{tabular}

Source : Données recueillies en 2017 sur les sites des établissements de langues et littératures romanes des universités polonaises.

Cette présentation sommaire des filières linguistiques reflète le premier cycle d'études (la licence), car celui de la maîtrise est plus complexe, souvent 
interdisciplinaire. De même, nous n'avons pas mentionné la différence du cursus selon la compétence langagière à l'admission : niveau avancé et niveau débutant, ce dernier avec un cours intensif du français pendant les deux premières années. Encore doit-on ajouter la spécialisation en didactique du français langue étrangère attestée par le diplôme de fin d'études. Afin de montrer toute la complexité des programmes d'études, un tour d'horizon plus détaillé reste encore à faire.

Récapitulons : Les établissements universitaires portant l'intitulé filologia romańska (philologie romane) ou romanistyka (études romanes) présentent des modes d'organisation différents.

Les six instituts ayant dans l'intitulé philologie romane (tableau 1) offrent des filières spécialisées pour le français et pour les autres langues romanes : leur liste va de deux (UG, KUL) jusqu'à cinq à l'Université Jagellonne. La diversité ne concerne que l'offre didactique de l'Institut, l'étudiant est contraint de choisir une seule filière. Des six établissements universitaires se réclamant de philologie romane, de néophilologie ou de langue et culture françaises (tableaux 2 et 3) trois, soit ceux de Opole, Szczecin et Zielona Góra ont des programmes d'études basés sur le français, tandis que ceux de Białystok et de Torun offrent des combinaisons du français avec une deuxième langue, romane ou autre. Les programmes de l'Université pédagogique (tableau 3) contiennent, en plus de la combinaison : philologie romane et une deuxième langue, deux filières autonomes : études hispaniques et italiennes.

Les études romanes (romanistyka) apparaissent dans l'intitulé des instituts universitaires de Łódź et de Varsovie (tableau 4). Les études romanes de Łódź se réalisent dans deux filières autonomes: française et italienne. Le programme de l'Institut d'études romanes de Varsovie se limite, par contre, aux seules études françaises, avec pour complément une deuxième langue (romane ou autre), tandis que l'italien, l'espagnol, le portugais sont enseignés comme première langue dans les instituts et chaires autonomes de la Faculté de néophilologie. Un autre établissement de la même université, l'Institut de linguistique appliquée (tableau 6), assure un enseignement centré sur les langues de spécialité et la traduction.

Une structure originale est créée à l'Institut des langues romanes et de traduction, Université de Silésie (tableau 5). Son programme, basé sur une combinaison de deux langues romanes avec l'anglais, est majoritairement centré sur les langues de spécialité et la traduction, ce qui permet de le qualifier comme appartenant au domaine des langues appliquées.

Ce bref examen montre à quel point les actuels programmes universitaires des langues romanes sont sélectifs et lacunaires par rapport à un domaine jadis unitaire de la philologie romane. Aujourd'hui celle-ci ne constitue plus une discipline homogène, mais une constellation unissant diverses branches des sciences humaines dont les recherches concernent un espace linguistique et culturel choisi.

Compte tenu des changements survenus ces dernières décennies, il serait tout à fait légitime de postuler un changement de l'étiquette philologie, collée 
traditionnellement aux établissements universitaires polonais spécialisés en langues romanes. Pourtant, un tel postulat se heurte inévitablement à un obstacle linguistique car, autant que les études italiennes/espagnoles/portugaises, etc., ont tout naturellement leurs équivalents polonais italianistyka, iberystyka, hispanistyka, portugalistyka, autant l'équivalent polonais des études françaises reste jusqu'à présent introuvable ${ }^{16}$. Une traduction littérale studia francuskie serait ambiguë, car elle signifierait aussi bien " études universitaires en France », ou « recherches en langue, littérature, culture françaises » que « études universitaires de langue, littérature, culture françaises ».

\section{UN DÉBAT RÉCENT SUR LA PHILOLOGIE UNIVERSITAIRE}

Ce débat fut organisé par la section des langues et lettres de la Société varsovienne des sciences le 15 mai $2017^{17}$. Les interventions concernaient différentes langues et lettres modernes enseignées dans les structures universitaires polonaises (les philologies et néophilologies) et étrangères, notamment celles en France, en Allemagne et en Italie. La thématique du débat et les conclusions méritent bien un bref résumé, car elles montrent à quel point la dénomination officielle de la discipline s'écarte de la réalité.

La question quelque peu provocatrice posée par Krzysztof Bogacki : «Peuton devenir philologue en étudiant la philologie romane au début du $\mathrm{XXI}^{\mathrm{e}}$ siècle ?» fondée sur une riche argumentation confrontant les exigences méthodologiques de cette discipline avec l'actuel cursus universitaire aboutit à une conclusion négative. À son avis, la philologie romane, telle qu'on l'enseigne encore dans les rares établissements universitaires en France, se tourne davantage vers la problématique socio-historique, tout en maintenant l'histoire de la langue et l'édition des textes. La philologie romane en Pologne, là où elle n'a pas complètement disparu du cursus, n'offre que des bribes de connaissances, largement insuffisantes pour entreprendre une recherche philologique. En conclusion donc, la philologie au sens propre n'entre plus de nos jours dans les compétences d'un diplômé polonais des langues et lettres modernes.

Le point de vue d'un philologue orientaliste montre une intéressante voie à suivre pour un renouvellement des programmes universitaires dans ce domaine. Dans son intervention intitulée «La philologie orientale a-t-elle encore un sens ? » Maria Krzysztof Byrski relate l'instauration d'un double parcours universitaire : la philologie au sens strict dont la vocation est l'étude des langue(s), littérature(s)

${ }^{16}$ Un dérivé par analogie : *francuzystyka, semble un barbarisme inacceptable pour la norme polonaise d'aujourd'hui. Une autre solution serait filologia francuska, tout aussi incommode.

${ }^{17}$ Les interventions sont publiées sur le site de la Société varsovienne des sciences (Towarzystwo Naukowe Warszawskie), Section I (Wydział I) : <http://www.tnw.waw.pl/index.php/konferencje-i> [consulté le 3.07.2018]. 
et civilisation(s) orientale(s) choisie(s) et, d'autre part, les études interculturelles qui permettent d'approcher les problèmes contemporains des pays asiatiques et africains. Tandis que la philologie orientale est une filière censée former des cadres universitaires, l'interculturel est une proposition adressée à un public plus large, à des personnes désireuses d'approfondir la connaissance de la civilisation d'une région du monde, pour des raisons professionnelles ou autres.

Deux cursus parallèles, la philologie et les études appliquées des langues modernes, sont une solution couramment adoptée dans la programmation des universités italiennes, ce qui a été le thème de l'intervention d'Elżbieta Jamrozik (« Glottologia, filologia, lingue moderne - la philologie dans l'enseignement universitaire des langues et lettres en Italie »). La tradition philologique italienne est basée sur le lycée classique dont le baccalauréat garantit une bonne connaissance du latin et du grec. Toutefois, les candidats qui se décident d'étudier les langues n'ont pas tous ce profil-là, d'où une dichotomie qui se dessine déjà au premier cycle universitaire entre les études appliquées où l'accent est mis sur l'acquisition pratique des langues modernes, et la philologie, réduite au minimum. Elle devient manifeste au niveau de la maîtrise qui offre, d'un côté, des matières utiles pour une future carrière professionnelle, telles la traduction spécialisée ou la glottodidactique, et de l'autre, un programme philologique qui exige une bonne connaissance des langues classiques et est adressé à une nette minorité.

La philologie polonaise jouit dans notre pays d'un statut spécial parmi les études des lettres et langues modernes, en tant que détentrice du patrimoine linguistique et littéraire national. Les auteurs Halina Karaś et Izabela WiniarskaGórska («Philologie dans le cursus des études polonaises »), tout en accentuant ce rôle important, présentent aussi les études polonaises appliquées qui contiennent de nouveaux éléments permettant de renouer un dialogue avec la modernité : sociolinguistique et psycholinguistique, didactique du polonais langue étrangère, logopédie, culture de la langue, édition, etc.

À l'étranger les études polonaises sont une branche de la slavistique. Alicja Nagórko (" Philologie polonaise en tant que philologie étrangère ») se réfère aux études slaves à l'Université Humboldt de Berlin, où celles-ci sont organisées en trois filières : littérature, linguistique et civilisation. Il est à noter que les départements allemands des langues modernes ont renoncé à l'intitulé de philologie, lui préférant celui de Slawistik, Germanistik, Orientalistik, Romanistik (Italianistik, Hispanistik, Lusitanistik...), etc. ; restent les départements d'études françaises pour lesquels un nom synthétique n'a pas pu être trouvé. En revenant aux études des langues et lettres modernes, elles sont regroupées (le polonais compris) dans la Faculté de linguistique et littérature (Sprach- und Literaturwissenschaftliche Fakultät) ; toutefois ce titre devient de moins en moins adapté au nouveau cursus à mesure que la composante culturelle du programme prend des dimensions toujours plus importantes.

Ce bref tour d'horizon des départements universitaires issus de la philologie amène à constater qu'il s'y est produit une rupture avec les contenus et les 
méthodes de cette discipline, traditionnellement vouée aux documents écrits et à leur langue. Ewa Geller («Philologie en tant que science de la communication ») note toutefois que c'est l'objet même, le logos, dans le nom de cette vieille discipline qui assure la continuité de la recherche. Le logos, la parole produisant le texte, doit être envisagé dans toutes ses dimensions : sociale, psychologique, cognitive, sémiotique, autrement dit, dans un fonctionnement complexe de la communication verbale. C'est donc la raison pour laquelle la philologie avec sa méthodologie spécifique d'approche du texte devrait bien trouver sa place dans le vaste domaine des sciences de la communication.

\section{CONCLUSION}

La philologie comme méthodologie de recherche n'a pas perdu tous ses atouts. Son approche diachronique peut être d'un grand secours dans l'étude de certains textes littéraires et documents écrits en général, ainsi que dans les recherches lexicales, historiques et cognitives. Par contre, les cursus actuels des études linguistiques, littéraires et civilisationnelles modernes gagneraient à abandonner l'étiquette contraignante de philologie car, conformément à la tendance actuelle, l'université doit avant tout aider les étudiants à acquérir des compétences utiles pour leur future vie professionnelle, aussi les matières enseignées relèvent-elles de plus en plus des sciences appliquées, tandis que les problèmes théoriques sont marginalisés.

Une séparation entre la philologie stricto sensu et les études appliquées des langues et lettres modernes est donc chose faite, il n'y a pas de retour en arrière. Il serait toutefois pour le moins imprudent, sinon irrationnel, de renoncer en bloc à l'immense richesse des résultats de recherches philologiques, d'ailleurs accessibles mieux que jamais grâce aux grandes bases de textes et documents numérisés. Dans les universités qui possèdent un encadrement didactique adéquat, on pourrait essayer de réintroduire les acquis de la philologie, en procédant à des remaniements du cursus. Par exemple, dans le premier cycle, on mettrait à la place du cours de grammaire historique (d'ailleurs déjà supprimé dans plusieurs départements de langues) un cours de lexicologie/sémantique à tendance historique, consistant dans l'étude des grands dictionnaires monolingues anciens et modernes, leur macrostructure, la structure de l'article et différentes formules de la définition. Dans le second cycle, serait optionnellement proposé un séminaire philologique relié à la spécialisation en traduction, en particulier des textes littéraires antérieurs au XIX ${ }^{\mathrm{e}}$ siècle. Les étudiants à ce niveau seraient certainement capables d'apprécier l'utilité du savoir philologique et de l'érudition, quelle que soit la carrière professionnelle à laquelle ils aspirent. 


\section{IS ROMANCE PHILOLOGY IN POLAND STILL WORTHY OF ITS NAME?}

\section{Summary}

The question discussed in this article is whether Romance philology as didactic matter is still present in Polish university curricula and does it really represent main Romance languages. Some departments of Romance philology have separate curricula for French, Italian or Spanish, the others teach only French. The current trend seems to prefer practical subjects like professional translation or teaching foreign languages rather than historical linguistics or serious literary studies. Nevertheless, a solution could be found to preserve philological profile at master degree courses, by creating an optional curriculum containing, for example, historical lexicology and lexicography as well as translation of ancient literature, especially medieval and renaissance works.

Key words: Romance philology, curricula, Polish university. 